\title{
Erratum: Epidemiology of lung cancer in Northern Greece: An 18-year hospital-based cohort study focused on the differences between smokers and non-smokers
}

\section{European Publishing Production Team}

By Kalliopi Domvri, Konstantinos Porpodis, Panagiota Zisi, Apostolos Apostolopoulos, Angeliki Cheva, Theodora Papamitsou, Despoina Papakosta, Theodoros Kontakiotis

Tobacco Induced Diseases, Volume 18, Issue March, Pages 1-8

Publish date: 24 March 2020

DOI: https://doi.org/10.18332/tid/118718

In the original article, in the first page, in the authors list, the superscripts indicating the authors' affiliations were mistakenly given as follows:

Kalliopi Domvri ${ }^{1,2}$, Konstantinos Porpodis ${ }^{1,2}$, Panagiota Zisi ${ }^{1,2}$, Apostolos Apostolopoulos $^{1,2}$, Angeliki Cheva ${ }^{3}$, Theodora Papamitsou ${ }^{4}$, Despoina Papakosta ${ }^{1,2}$, Theodoros Kontakiotis ${ }^{1,2}$

The superscripts in the original manuscript are now corrected:

Kalliopi Domvri ${ }^{1}$, Konstantinos Porpodis ${ }^{1}$, Panagiota Zisi ${ }^{1}$, Apostolos Apostolopoulos ${ }^{1}$, Angeliki Cheva ${ }^{2}$, Theodora Papamitsou ${ }^{3}$, Despoina Papakosta ${ }^{1}$, Theodoros Kontakiotis ${ }^{1}$

${ }^{1}$ Department of Respiratory Medicine, Aristotle University of Thessaloniki, George Papanikolaou Hospital, Thessaloniki, Greece

${ }^{2}$ Laboratory of Pathology, Medical School, Aristotle University of Thessaloniki, Thessaloniki, Greece

${ }^{3}$ Laboratory of Histology-Embryology, Aristotle University of Thessaloniki, Thessaloniki, Greece

\section{AFFILIATION \\ 1 European Publishing, \\ Heraklion, Greece \\ CORRESPONDENCE TO European Publishing \\ Production Team. \\ Office 108, C Building, \\ Science and Technology \\ Park of Crete (STEP-C) \\ Vassilika Vouton, GR- \\ 70013 Heraklion, Crete, \\ Greece. E-mail: info@ \\ europeanpublishing.eu \\ KEYWORDS \\ lung cancer, epidemiology, \\ smoking, bronchoscopy, \\ histology}

Received: 13 April 2020

Accepted: 13 April 2020 